\title{
Clinically Feasible Microstructural MRI to Quantify Cervical Spinal Cord Tissue Injury Using DTI, MT, and T2*-Weighted Imaging: Assessment of Normative Data and Reliability
}

(D) A.R. Martin, (DB. De Leener, (D). Cohen-Adad, (DD.W. Cadotte, (D) S. Kalsi-Ryan, DS.F. Lange, (D) Tetreault, (D) A. Nouri, (D) A. Crawley, DD.J. Mikulis, (1) H. Ginsberg, and (DM.G. Fehlings

\begin{abstract}
BACKGROUND AND PURPOSE: DTI, magnetization transfer, $\mathrm{T} 2{ }^{*}$-weighted imaging, and cross-sectional area can quantify aspects of spinal cord microstructure. However, clinical adoption remains elusive due to complex acquisitions, cumbersome analysis, limited reliability, and wide ranges of normal values. We propose a simple multiparametric protocol with automated analysis and report normative data, analysis of confounding variables, and reliability.
\end{abstract}

MATERIALS AND METHODS: Forty healthy subjects underwent T2WI, DTI, magnetization transfer, and T2*WI at $3 \mathrm{~T}$ in $<35$ minutes using standard hardware and pulse sequences. Cross-sectional area, fractional anisotropy, magnetization transfer ratio, and T2*WI WM/GM signal intensity ratio were calculated. Relationships between MR imaging metrics and age, sex, height, weight, cervical cord length, and rostrocaudal level were analyzed. Test-retest coefficient of variation measured reliability in 24 DTI, 17 magnetization transfer, and 16 T2*WI datasets. DTI with and without cardiac triggering was compared in 10 subjects.

RESULTS: T2*WI WM/GM showed lower intersubject coefficient of variation $(3.5 \%)$ compared with magnetization transfer ratio (5.8\%), fractional anisotropy (6.0\%), and cross-sectional area (12.2\%). Linear correction of cross-sectional area with cervical cord length, fractional anisotropy with age, and magnetization transfer ratio with age and height led to decreased coefficients of variation (4.8\%, 5.4\%, and $10.2 \%$, respectively). Acceptable reliability was achieved for all metrics/levels (test-retest coefficient of variation $<$ $5 \%$ ), with T2*WI WM/GM comparing favorably with fractional anisotropy and magnetization transfer ratio. DTI with and without cardiac triggering showed no significant differences for fractional anisotropy and test-retest coefficient of variation.

CONCLUSIONS: Reliable multiparametric assessment of spinal cord microstructure is possible by using clinically suitable methods. These results establish normalization procedures and pave the way for clinical studies, with the potential for improving diagnostics, objectively monitoring disease progression, and predicting outcomes in spinal pathologies.

ABBREVIATIONS: $\mathrm{CSA}=$ cross-sectional area; $\mathrm{DCM}=$ degenerative cervical myelopathy; $\mathrm{FA}=$ fractional anisotropy; $\mathrm{MCL}=$ maximally compressed level; $\mathrm{MT}=$ magnetization transfer; $\mathrm{MTR}=$ magnetization transfer ratio; $\mathrm{SC}=$ spinal cord; $\mathrm{TRCOV}=$ test-retest coefficient of variation

$\mathbf{T}$ he era of quantitative MR imaging has arrived, allowing in vivo measurement of specific physical properties reflecting spinal cord (SC) microstructure and tissue damage. ${ }^{1,2}$ Such measures have potential clinical applications, including improved di-

Received October 20, 2016; accepted after revision January 28, 2017.

From the Division of Neurosurgery, Department of Surgery (A.R.M., D.W.C., S.K.-R., L.T., A.N., H.G., M.G.F.), and Department of Medical Imaging (A.C., D.J.M.), University of Toronto and the University Health Network, Toronto Western Hospital, Toronto, Ontario, Canada; Polytechnique Montreal (B.D.L., J.C.-A.), Montréal, Quebec, Canada; Functional Neuroimaging Unit (J.C.-A.), Centre de recherche de l'Institut universitaire de gériatrie de Montréal, Université de Montréal, Montréal, Quebec, Canada; and University of Groningen (S.F.L.), Groningen, the Netherlands.

Please address correspondence to Michael G. Fehlings, MD, SCI-CRU, 11th Floor, Toronto Western Hospital, 399 Bathurst St, Toronto, M5T 2S8, ON, Canada; e-mail: michael.fehlings@uhn.on.ca, madeleineoh@gmail.com; @DrFehlings

- Indicates open access to non-subscribers at www.ajnr.org

http://dx.doi.org/10.3174/ajnr.A5163 agnostic tools, objective monitoring for disease progression, and prediction of clinical outcomes. ${ }^{3}$ However, technical challenges such as artifacts, image distortion, and achieving acceptable SNR have led to limited reliability. Specialized pulse sequences and custom hardware have advanced the field but incur costs of increased complexity and acquisition time while creating barriers to portability and clinical adoption. Furthermore, quantitative MR imaging metrics often show wide ranges of normal values and confounding relationships with subject characteristics such as age, ${ }^{4-8}$ for which most previous studies have not accounted. ${ }^{3}$

Among the most promising SC quantitative MR imaging techniques are DTI and magnetization transfer (MT). ${ }^{1-3}$ These provide measures of axonal integrity and myelin quantity that correlate with functional impairment in conditions such as degenerative cervical myelopathy (DCM $)^{5-7,9}$ and $\mathrm{MS},{ }^{3,9}$ albeit with 
Table 1: Acquisition protocol ${ }^{\mathrm{a}}$

\begin{tabular}{|c|c|c|c|c|}
\hline Imaging Type & $\begin{array}{l}\text { Pulse Sequence; } \\
\text { Orientation }\end{array}$ & Technical Details & Acquisition Time & Metric \\
\hline $\mathrm{T} 2 \mathrm{WI}$ & 3D FIESTA-C; sagittal & $\begin{array}{l}\mathrm{TR} / \mathrm{TE}=5.4 / 2.6 \mathrm{~s}, \mathrm{FOV}=200 \times 200 \mathrm{~mm}^{2}, \\
\text { matrix }=256 \times 256, \text { resolution }=0.8 \times 0.8 \times \\
0.8 \mathrm{~mm}^{3}, \mathrm{NEX}=2 \text {, flip angle }=35^{\circ}\end{array}$ & $6 \min 56 s$ & CSA \\
\hline DTI & $\begin{array}{l}\text { Spin-echo ssEPI with } \\
\text { OVS; axial }\end{array}$ & $\begin{array}{l}\mathrm{TR} / \mathrm{TE}=4050 / 91.2 \mathrm{~ms}, \mathrm{FOV}=80 \times 80 \mathrm{~mm}^{2} \\
\text { matrix }=64 \times 64, \text { resolution }=1.25 \times \\
1.25 \times 5 \mathrm{~mm}^{3}, 25 \text { directions }\left(b=800 \mathrm{~s} / \mathrm{mm}^{2}\right) \\
5 b=0 \mathrm{~s} / \mathrm{mm}^{2} \text { images, AP saturation bands, } \\
\text { phase encoding }=\mathrm{AP}, 2 \text { 2nd-order shimming }\end{array}$ & $\begin{array}{l}3 \times 2 \min 6 s, 1 \min 30 \mathrm{~s} \\
\text { for shimming }\end{array}$ & FA \\
\hline MT & $\begin{array}{l}\text { 2D SPGR with/without } \\
\text { prepulse; axial }\end{array}$ & $\begin{array}{l}\mathrm{TR} / \mathrm{TE}=32 / 5.9 \mathrm{~ms}, \mathrm{FOV}=190 \times 190 \mathrm{~mm}^{2}, \\
\text { matrix }=192 \times 192, \text { resolution }=1 \times 1 \times \\
5 \mathrm{~mm}^{3}, \mathrm{NEX}=3 \text {, flip angle }=6^{\circ}, \text { flow } \\
\text { compensation, phase encoding }=\mathrm{AP}, \\
\text { prepulse: Gaussian, duration }=9984 \mu \mathrm{s}, \\
\text { offset }=1200 \mathrm{~Hz}\end{array}$ & $\begin{array}{l}3 \text { min } 45 \mathrm{~s} \text { each, with and } \\
\text { without prepulse }\end{array}$ & MTR \\
\hline $\mathrm{T} 2 * \mathrm{WI}$ & 2D MERGE; axial & $\begin{array}{l}\mathrm{TR} / \mathrm{TE}=650 / 5,10,15 \mathrm{~ms}, \mathrm{FOV}=200 \times \\
200 \mathrm{~mm}^{2}, \text { matrix }=320 \times 320 \text {, resolution }= \\
0.6 \times 0.6 \times 4 \mathrm{~mm}^{3}, \mathrm{NEX}=1 \text {, flip angle }= \\
20^{\circ}, \mathrm{BW}=62 \mathrm{kHz} \text { per line }\end{array}$ & $3 \min 33 s$ & WM/GM ratio \\
\hline
\end{tabular}

Note:-AP indicates anteroposterior; BW, bandwidth; FIESTA-C, FIESTA-cycled phases; MERGE, multiecho recombined gradient echo; OVS, outer volume suppression; SPGR, echo-spoiled gradient echo; ssEPI, single-shot echo-planar imaging.

${ }^{a}$ Technical specifications of our multiparametric cervical SC MRI protocol, with an acquisition time of 25 minutes (30-35 minutes, including positioning, section prescription, shimming, and prescans).

limited physiologic specificity (eg, fractional anisotropy [FA] reflects both demyelination and axonal injury). ${ }^{10,11}$ SC crosssectional area (CSA) computed from high-resolution anatomic images can measure atrophy (eg, in MS $)^{12}$ or the degree of SC compression in DCM. ${ }^{13} \mathrm{~T}^{*}$-weighted imaging at $3 \mathrm{~T}$ or higher field strengths offers high resolution and sharp contrast between SC WM and GM, allowing segmentation between these structures similar to that in phase-sensitive inversion recovery. ${ }^{14,15} \mathrm{~T} 2{ }^{*} \mathrm{WI}$ also demonstrates hyperintensity in injured $\mathrm{WM},{ }^{16-18}$ reflecting demyelination, gliosis, and increased calcium and nonheme iron concentrations. ${ }^{19} \mathrm{~T} 2{ }^{*} \mathrm{WI}$ signal intensity is not an absolute quantity, so we normalize its value in WM by the average GM signal intensity in each axial section, creating a novel measure of WM injury: T2*WI WM/GM ratio. ${ }^{20}$

We propose a multiparametric approach to cervical SC quantitative MR imaging with clinically feasible methods, including acceptable acquisition times, standard hardware/pulse sequences, and automated image analysis. Our protocol yields 4 measures of SC tissue injury (CSA, FA, MT ratio [MTR], and T2*WI WM/GM), for which this study establishes normative values in numerous ROIs. We characterize the variation of these metrics with age, sex, height, weight, cervical cord length, and rostrocaudal level and propose normalization methods. Finally, we assess testretest reliability of FA, MTR, and T2*WI WM/GM and compare our DTI results against those with cardiac triggering.

\section{MATERIALS AND METHODS \\ Study Design and Subjects}

This study received approval from the University Health Network (Toronto, Ontario, Canada), and written informed consent was obtained from all participants. Forty-two subjects were recruited between October 2014 and December 2016 with a broad range of ages and balance between sexes. A physician (A.R.M.) assessed all subjects to rule out symptoms and signs of neurologic dysfunction, and T2WI was screened for abnormalities suggestive of mul- tiple sclerosis, tumor, or severe cord compression. Two subjects were excluded from the study with clinical and imaging findings of DCM, leaving 40 healthy subjects for analysis. Data from 18 patients with DCM were included for analysis of test-retest reliability, and 6 patients with DCM were included in a cardiac-triggering comparison, but subjects with DCM were excluded from other analyses. $^{20}$

\section{MR Imaging Acquisitions}

MR images were acquired on a 3T clinical scanner (Signa Excite HDxt; GE Healthcare, Milwaukee, Wisconsin). Peak gradients were $50 \mathrm{mT} / \mathrm{m}$; slew rate, $150 \mathrm{~T} / \mathrm{m} / \mathrm{s}$ with a body coil for transmission and the top 2 elements of a standard 8-element spine coil (Premier III Phased Array CTL; USA Instruments, Aurora, Ohio) for reception. Subjects were positioned head-first and supine with the head tightly padded to prevent movement and the neck flexed to straighten the cervical SC.

The MR imaging protocol was developed on the basis of methods previously used by one of the authors (J.C.-A.). ${ }^{16,17,21}$ T2WIs used sagittal FIESTA-cycled phases with $0.8-\mathrm{mm}^{3}$ isotropic resolution covering the brain stem to T4. DTI, MT, and T2*WI had 13 axial sections positioned perpendicular to the spinal cord (at C3), covering $\mathrm{C} 1-\mathrm{C} 7$ by using a variable gap, alternating between the mid-vertebral body and the intervertebral disc. Parameters for each sequence are listed in Table 1. DTI used a spin-echo singleshot EPI sequence with an $80 \times 80 \mathrm{~mm}^{2}$ FOV to minimize susceptibility distortions, anterior/posterior saturation bands to achieve outer volume suppression, and no cardiac triggering. Second-order localized shimming was performed before DTI by positioning a VOI encompassing the SC from C1-C7. T2*WIs used the multiecho recombined gradient-echo sequence, with 3 echoes that are magnitude-reconstructed and combined by using a sumof-squares algorithm. ${ }^{18}$ Each session required 30-35 minutes, including subject positioning, section prescription, prescanning, and shimming. 


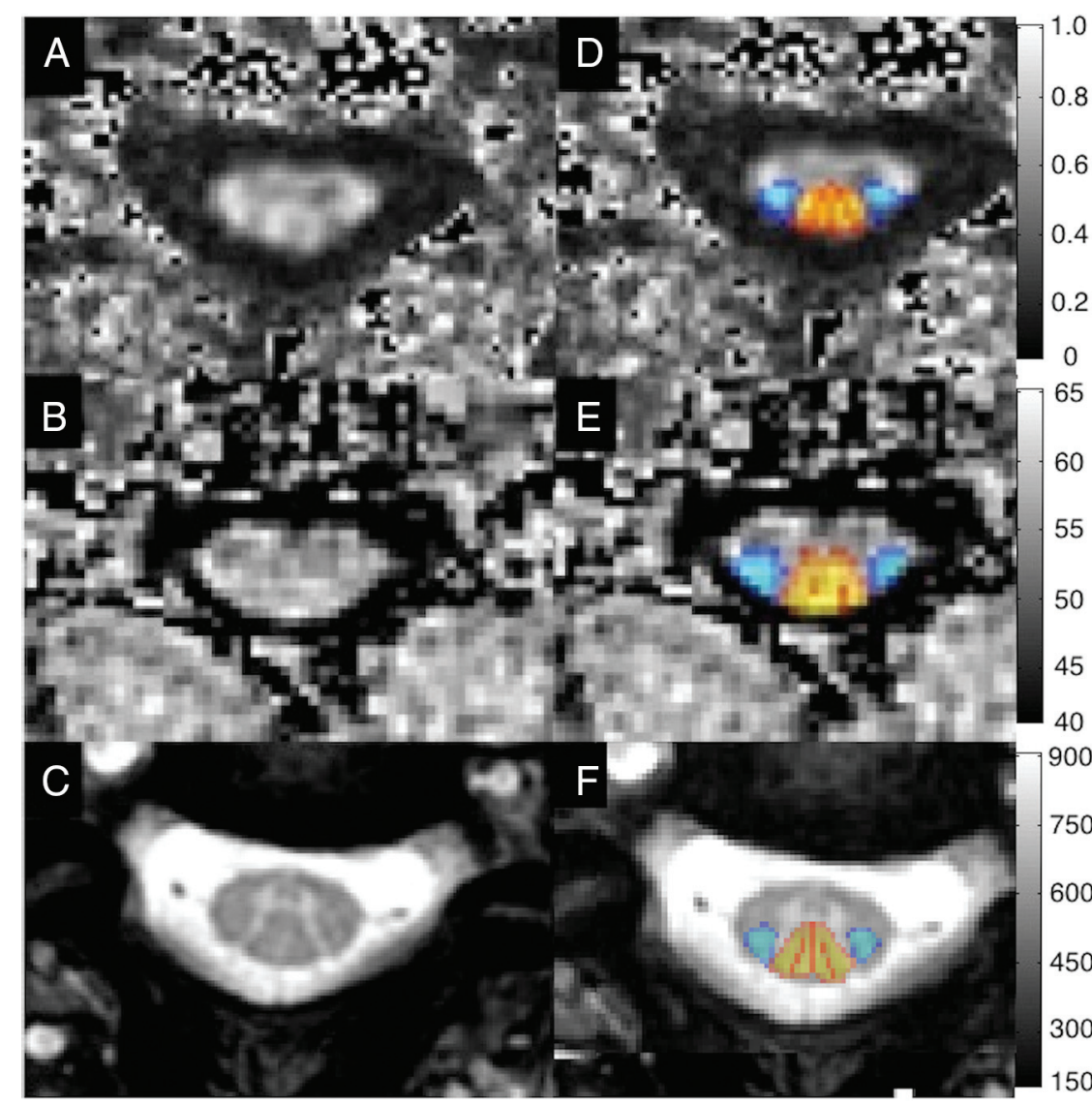

FIG 1. Representative images showing FA maps $(A)$, MTR maps $(B)$, and $T 2^{*} \mathrm{WI}(C)$ with probabilistic maps of the lateral corticospinal tracts (blue) and dorsal columns (red-yellow) overlaid $(D-F)$ following registration to the SCT atlas. iting. Images were nonlinearly registered to the MNI-Poly-AMU template/ atlas in SCT. ${ }^{23}$ T2WIs were used to automatically calculate cervical cord length (from the top of $\mathrm{C} 1$ to the bottom of the C7 vertebral levels) and SC CSA. DTI was motion-corrected with regularized registration, and diffusion tensors were calculated with outlier rejection by using the RESTORE (robust estimation of tensors by outlier rejection) method. ${ }^{24}$ MT images with and without prepulses were coregistered, and MTR was computed. T2*WI data were further analyzed with automatic segmentation of GM and $\mathrm{WM},{ }^{25}$ which was used to refine the registration of $\mathrm{T} 2{ }^{\star} \mathrm{WI}$ to the template. FA, MTR, and T2*WI WM/GM ratios were extracted from various ROIs by using the SCT probabilistic atlas with automatic correction for partial volume effects by using the maximum a posteriori method. ${ }^{26}$ ROIs included the SC, $\mathrm{WM}$, and GM and the left/right lateral corticospinal tract, fasciculus cuneatus, fasciculus gracilis, and spinal lemniscus in each axial section (Fig 1). Metrics were averaged at rostral $(\mathrm{C} 1-\mathrm{C} 3)$, middle (C4-5) or maximally compressed (MCL, subjects with DCM), and caudal (C6-C7) levels.
Test-retest reliability was assessed by removing the subject from the scanner and repositioning before rescanning. This was performed in a subset of subjects (DTI: 17 healthy, 9 with DCM; MT: 13 healthy, 4 with DCM; T2*WI: 5 healthy, 11 with DCM) extemporaneously, depending on scanner availability and subject willingness. Reliability was not assessed for SC CSA measurement due to time constraints.

A comparison of DTI with and without cardiac triggering was also performed in 10 subjects ( 4 healthy, 6 with DCM). Cardiac-triggered DTI was performed with pulse oximetry triggering, trigger delay of $310 \mathrm{~ms}$, window of $250 \mathrm{~ms}$, and $\mathrm{TR}=7 \mathrm{R}-\mathrm{R}$ interval. Two acquisitions were performed that were analyzed individually for test-retest coefficient of variation (TRCOV) and then concatenated and averaged for comparison with nontriggered DTI.

\section{Image Analysis Techniques}

Imaging data were analyzed by using the Spinal Cord Toolbox, Version2.3 (SCT; https://www.nitrc.org/projects/sct/). ${ }^{22}$ Each axial image was visually inspected by 1 rater (A.R.M.) and excluded if low signal or artifacts (motion, aliasing) were present. SC segmentation was automatically performed by using native T2WIs and T2*WIs, the mean diffusivity map for DTI, and the MT image with a prepulse. Segmentation errors were resolved by providing seed points for automatic segmentation or manual ed-

\section{Statistical Analysis}

Statistical analysis was performed with $\mathrm{R}$ statistical and computing software, Version 3.3 (http://www.r-project.org/). Normative data were summarized with mean, SD, and intersubject coefficient of variation. Relationships between MR imaging metrics (averaged from $\mathrm{C} 1-\mathrm{C} 7$ ) and patient characteristics (age, sex, height, weight, cervical cord length) were assessed with Pearson correlation coefficients and backward stepwise linear regression to determine significant independent relationships and their coefficients. Differences by rostrocaudal level were assessed with ANOVA. If differences were found, we calculated Spearman coefficients (between mean values and numbered levels) to identify monotonic relationships. To determine whether nonlinear relationships were present, we performed a likelihood ratio test on linear regression models with and without a 5-knot restricted cubic spline. Paired $t$ tests compared WM and GM differences, and ANOVA was used to identify differences among individual WM tracts (averaged bilaterally). Reliability was assessed by using testretest coefficient of variation, and differences between healthy subjects and those with DCM were assessed with Welch $t$ tests, as were pair-wise comparisons between techniques at each rostrocaudal level. Statistical significance was set to $P=.05$ and was not corrected for multiple comparisons due to the exploratory nature of this study.

AJNR Am J Neuroradiol 38:1257-65 Jun 2017 www.ajnr.org 
Table 2: Subject characteristics ${ }^{\mathrm{a}}$

\begin{tabular}{lcc}
\hline \multicolumn{1}{c}{ Characteristic } & Healthy Subjects $(\boldsymbol{n}=\mathbf{4 0})$ & Subjects with DCM $(\boldsymbol{n}=\mathbf{1 8})$ \\
\hline Age $(\mathrm{yr})$ & $47.1 \pm 15.3$ (range, 19-79) & $56.4 \pm 11.0$ (range, 36-76) \\
Sex & 21 men, 19 women & 11 men, 7 women \\
Height $(\mathrm{cm})$ & $171.4 \pm 8.6$ & $172.8 \pm 8.9$ \\
Weight $(\mathrm{kg})$ & $74.6 \pm 11.5$ & $79.0 \pm 15.1$ \\
Cervical cord length $(\mathrm{cm})$ & $10.6 \pm 1.0$ & $11.1 \pm 0.9$ \\
\hline
\end{tabular}

${ }^{a}$ Demographics and characteristics of 40 healthy subjects and 18 with DCM are shown. Data (other than sex) are reported as mean $\pm \mathrm{SD}$.

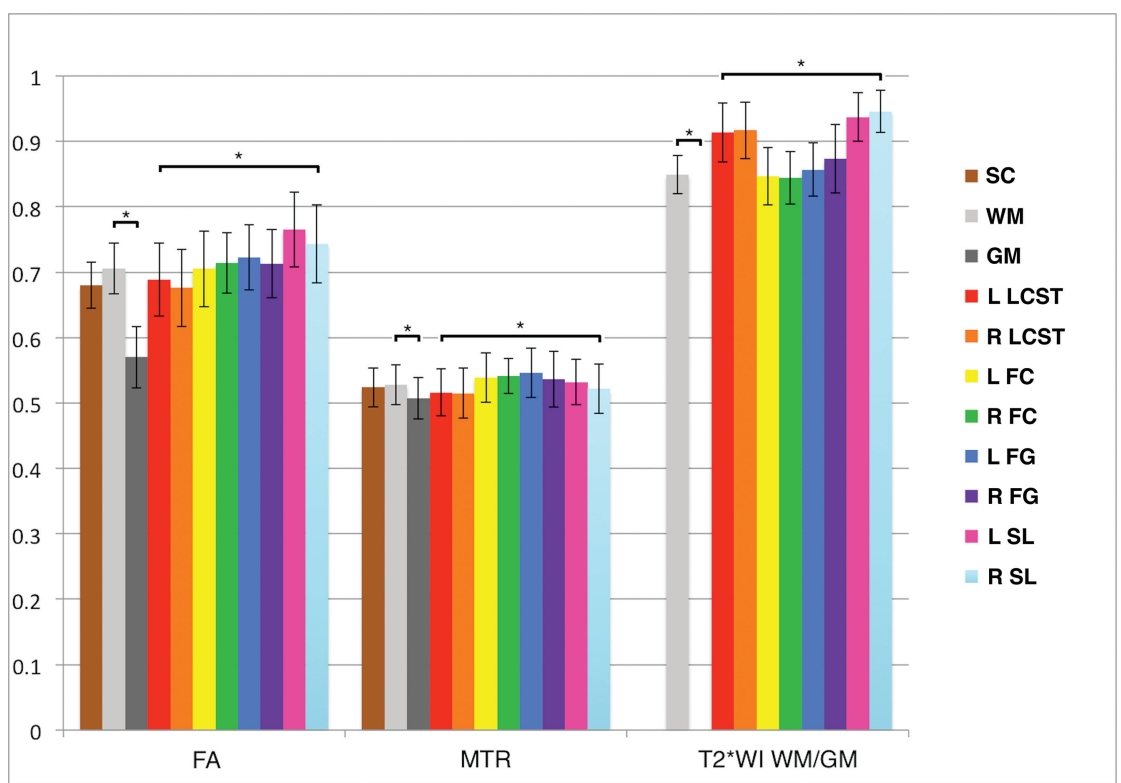

FIG 2. Normative data in the rostral cervical cord for FA, MTR, and T2*WI WM/GM ratios. Metrics are extracted from SC, WM, GM, and key WM tracts averaged over rostral sections (Cl-C3). Values are displayed as mean \pm intersubject SD (error bars). The asterisk denotes $P<.05$ with paired $t$ tests between WM and GM and ANOVA among WM tracts. L indicates left; R, right; FC, fasciculus cuneatus; FG, fasciculus gracilis; SL, spinal lemniscus; LCST, lateral corticospinal tract.

\section{RESULTS}

\section{Subject Characteristics}

Characteristics of 40 healthy subjects and 18 with DCM included in this study are listed in Table 2.

\section{Image Acquisition}

Acceptable image quality was achieved in all subjects and techniques. For DTI, 27 of 520 axial images $(5.2 \%)$ were excluded due to artifacts or poor signal. For MT and T2*WI, $6(1.2 \%)$ and 4 $(0.8 \%)$ sections were excluded due to artifacts, respectively.

\section{Automated Analysis}

Automated segmentation was frequently successful, with manual editing required in $8 \mathrm{~T} 2 \mathrm{WI}$ datasets (20\%), 14 MT datasets (35\%), 4 DTI datasets (10\%), and $20 \mathrm{~T} 2{ }^{\star} \mathrm{WI}$ datasets (50\%). Manual segmentation editing was usually restricted to a small number of sections and required $<5$ minutes per dataset. Automatic registration to the template and data extraction were successful in all cases.

\section{Normative Values for MR Imaging Metrics}

Normative data extracted from C1-C3 showed that T2*WI $\mathrm{WM} / \mathrm{GM}$ had the smallest intersubject coefficient of variation at $3.5 \%(0.848 \pm 0.028)$, compared with $5.8 \%$ for MTR $(52.8 \pm$ $3.1 \%), 6.0 \%$ for FA $(0.706 \pm 0.042)$, and $12.2 \%$ for CSA $(78.5 \pm$
$9.6 \mathrm{~mm}^{2}$ ) (Fig 2). The strongest contrast between WM and GM was found for $\mathrm{T} 2{ }^{\star} \mathrm{WI}$ signal intensity (mean GM-WM difference \pm standard error $=83.9 \pm$ 4.72, $\left.P=3 \times 10^{-20}\right)$, which exceeded that of FA $(-0.110 \pm 0.0083, P=2 \times$ $\left.10^{-15}\right)$ and MTR $(-2.1 \pm 0.28, P=4 \times$ $\left.10^{-9}\right)$. Individual WM tracts showed significant variations for $\mathrm{T}^{\star}{ }^{\star} \mathrm{WI}$ WM/GM (ANOVA, $P=2 \times 10^{-9}$ ), FA $\left(P=3 \times 10^{-7}\right)$, and $\operatorname{MTR}(P=.01)$.

\section{Variations with Subject Characteristics}

Univariate relationships between MR imaging metrics and subject characteristics included the following: CSA increased with cervical cord length $(P=$ $\left.8 \times 10^{-4}\right)$, weight $(P=.03)$, and male sex $(P=.03)$; FA decreased with age $(P=.009)$; and MTR decreased with height $(P=.008)$, weight $(P=.01)$, and male sex $(P=.006)$ (Table 3$)$. Trends were also present for CSA, increasing with height $(P=.06)$, and for $\mathrm{T} 2{ }^{\star} \mathrm{WI}$ WM/GM, increasing with age $(P=.06)$ and weight $(P=.06)$. In multivariate analysis, CSA varied only with cervical cord length $(\beta=+5.3690)$; FA, with age $(\beta=-0.0012053)$; and MTR, with height $(\beta=-0.17410, P=.001)$ and age $(\beta=-0.074131, P=.01)$, while $\mathrm{T} 2{ }^{*} \mathrm{WI} W M / \mathrm{GM}$ did not require normalization. Following linear corrections, intersubject coefficient of variation decreased to $4.8 \%$ for MTR, $5.4 \%$ for FA, and $10.2 \%$ for CSA.

\section{Metrics by Rostrocaudal Level}

ANOVA detected significant differences $(P<.05)$ across rostrocaudal levels for all metrics. Monotonic variations were present $(P<.05)$ for MTR $(\rho=-0.98)$, FA $(\rho=-0.90)$, and CSA $(\rho=$ $-0.55)$, which all decreased from rostral to caudal levels, whereas $\mathrm{T} 2{ }^{\star} \mathrm{WI} W M / G M$ showed a trend toward increasing $(\rho=0.53, P=$ .06) (Fig 3). CSA, FA, and T2*WI WM/GM showed nonlinear rostrocaudal variation $(P<.05)$, whereas MTR did not $(P=.58)$.

\section{Reliability}

The T2*WI WM/GM ratio was the most reliable metric (pooled TRCOV: rostral, 0.9\%; MCL, 2.9\%; caudal, 2.6\%), comparing favorably with FA (rostral, 2.6\%; MCL, 3.6\%; caudal, 3.2\%) and MTR (rostral, 2.4\%; MCL, 3.7\%; caudal: $4.2 \%$ ), though these differences were only significant for rostral metrics $(P<.05)$ (Table 4). Reliability measures were comparable between healthy subjects and those with DCM rostrally (C1-C3), but subjects with DCM trended toward increased TRCOV for MCL MTR (6.1\% versus $3.2 \%, P=.08)$ and caudal FA ( $4.6 \%$ versus $2.2 \%, P=.07)$. The reliability of data from individual WM tracts was acceptable 
Table 3: Univariate relationships of MRI metrics with healthy subject characteristics ${ }^{\mathrm{a}}$

\begin{tabular}{llclll}
\hline \multicolumn{1}{c}{ Metric } & \multicolumn{1}{c}{ Age } & Sex (M vs F) & Height & Weight \\
\hline CSA $\left(\mathrm{mm}^{2}\right)$ & $r=-0.25(P=.12)$ & $80.0 \pm 11.2$ vs $73.5 \pm 8.5\left(P=.03^{\mathrm{b}}\right)$ & $r=0.31\left(P=.06^{\mathrm{c}}\right)$ & $r=0.34\left(P=.03^{\mathrm{b}}\right)$ & $r=0.51\left(P<.001^{\mathrm{b}}\right)$ \\
FA & $r=-0.43\left(P=.009^{\mathrm{b}}\right)$ & $0.658 \pm 0.037$ vs $0.663 \pm 0.034(P=.75)$ & $r=-0.02(P=.89)$ & $r=-0.26(P=.12)$ & $r=0.11(P=.53)$ \\
MTR & $r=-0.25(P=.11)$ & $48.8 \pm 2.5$ vs $51.4 \pm 2.7\left(P=.006^{\mathrm{b}}\right)$ & $r=-0.41\left(P=.008^{\mathrm{b}}\right)$ & $r=-0.40(P=.01)$ & $r=-0.18(P=.26)$ \\
T2*WI WM/GM & $r=0.31(P=.06)$ & $0.863 \pm 0.034$ vs $0.858 \pm 0.031(P=.64)$ & $r=-0.12(P=.48)$ & $r=0.31\left(P=.06^{\mathrm{c}}\right)$ & $r=-0.09(P=.55)$ \\
\hline
\end{tabular}

a Values for sex are reported as mean \pm SD, and other values are Pearson correlation coefficient. FA, MTR, and T2*WI WM/GM ratios are extracted from WM, while CSA of the spinal cord is measured, averaged across $\mathrm{Cl}-\mathrm{C} 7$.

bignificance $(P<.05)$.

c Trends $(P<.10)$.

CSA vs. Vertebral Level

ANOVA: $p<0.001$ Spearman rho $=-0.55, p=0.05$

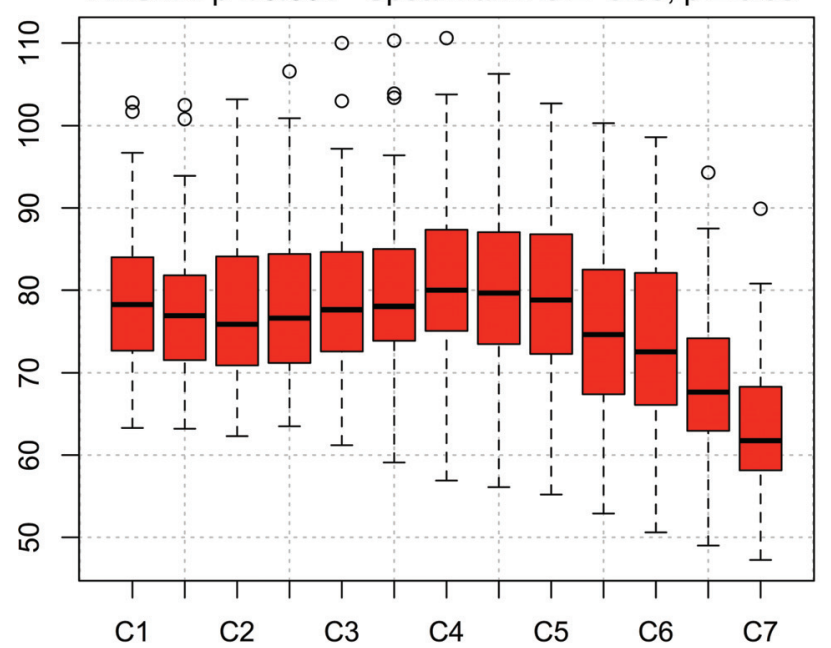

MTR vs. Vertebral Level

ANOVA: $p<0.001$ Spearman rho $=-0.98, p<0.001$

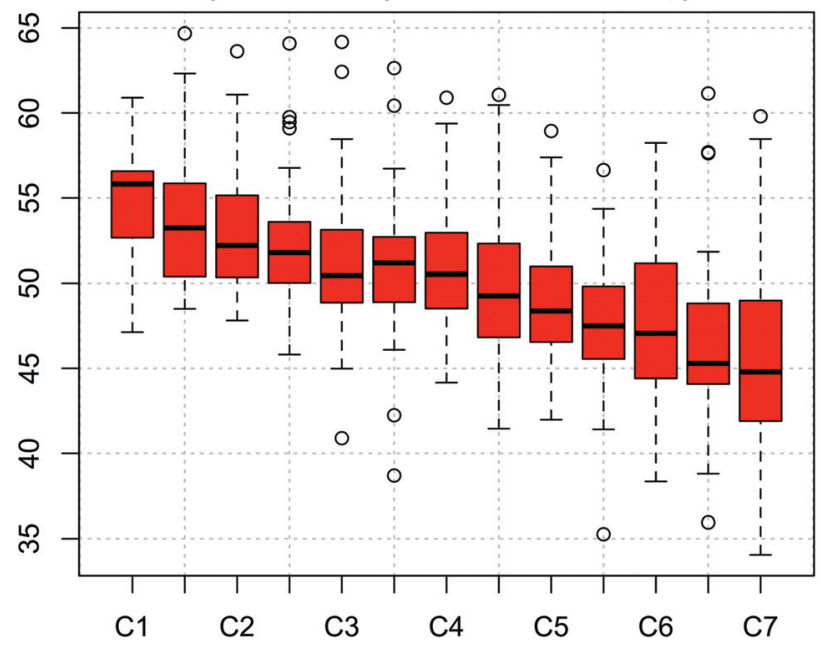

FA vs. Vertebral Level ANOVA: $p<0.001$ Spearman rho $=-0.90, p<0.001$

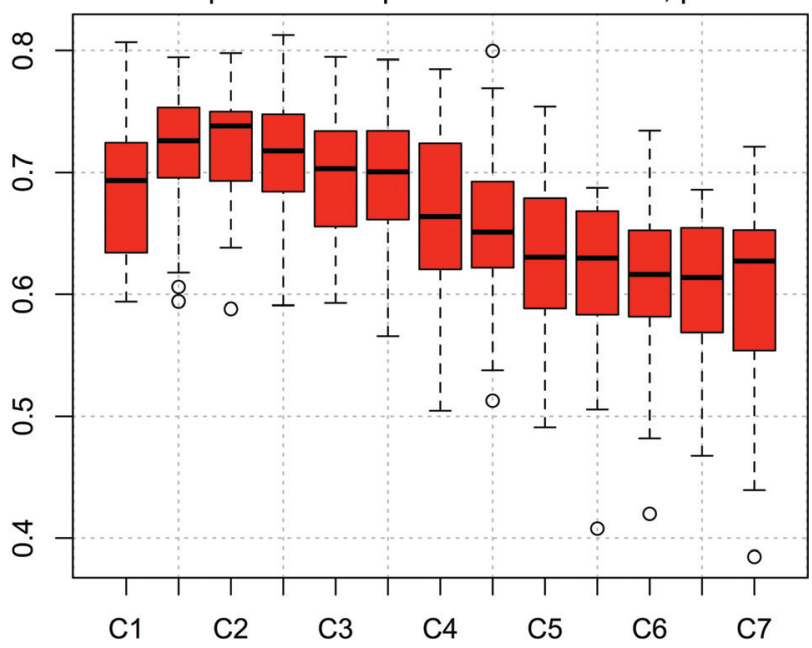

T2*WI WM/GM vs. Vertebral Level ANOVA: $p<0.001$ Spearman $r h o=0.53, p=0.06$

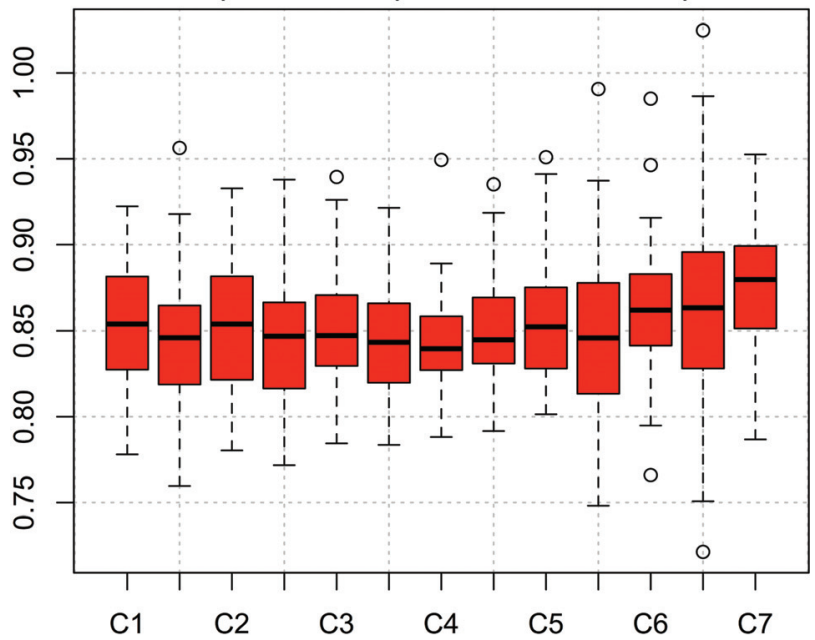

FIG 3. Variations by rostrocaudal level. MR imaging metrics displayed for each vertebral and intervertebral level from $\mathrm{Cl}$ to $\mathrm{C7}$. FA, MTR, and T2*WI WM/GM ratios are extracted from WM. ANOVA shows significant differences by level for all metrics. Monotonic variations are present for CSA, FA, and MTR.

(TRCOV $<5 \%$ ) except for FA of the right and left spinal lemniscus $(5.3 \%, 5.6 \%$, respectively; Fig 4$)$.

\section{Cardiac Triggering in DTI}

FA did not differ significantly among DTI acquisitions with and without cardiac triggering, though triggering showed a trend toward higher FA at MCL $(0.558$ versus $0.514, P=.06)$ and caudal ( 0.562 versus $0.534, P=.07$ ) levels (Table 5 ). No significant differences in TRCOV were observed, though cardiac-triggered DTI provided approximately $1 \%$ lower TRCOV at all levels.

\section{DISCUSSION}

\section{Summary of Findings}

This study establishes a multiparametric MR imaging protocol and analysis framework to assess the microstructure of the entire cervical SC by using simple methods that are feasible for clinical adoption, requiring only 20 minutes of acquisition 
Table 4: Test-retest reliability across rostrocaudal levels ${ }^{a}$

\begin{tabular}{clcccc}
\hline Level & \multicolumn{1}{c}{ Metric } & Healthy & DCM & P Value & Pooled \\
\hline Rostral (Cl-C3) & FA & $2.5 \pm 2.0 \%$ & $2.8 \pm 1.8 \%$ & .71 & $2.6 \pm 1.9 \%$ \\
& MTR & $2.7 \pm 1.9 \%$ & $1.3 \pm 0.5 \%$ & .17 & $2.4 \pm 1.9 \%$ \\
& T2*WI WM/GM & $0.9 \pm 0.6 \%$ & $1.0 \pm 0.7 \%$ & .77 & $0.9 \pm 0.7 \%$ \\
Midcervical (C4-C5) & FA & $3.0 \pm 2.2 \%$ & $5.0 \pm 5.7 \%$ & .21 & $3.6 \pm 3.6 \%$ \\
or MCL & MTR & $3.2 \pm 3.0 \%$ & $6.1 \pm 0.9 \%$ & $.08^{\mathrm{c}}$ & $3.7 \pm 3.2 \%$ \\
& T2*WI WM/GM & $1.4 \pm 1.1 \%$ & $3.5 \pm 2.2 \%$ & .11 & $2.9 \pm 2.2 \%$ \\
Caudal (C6-C7) & FA & $2.2 \pm 1.6 \%$ & $4.6 \pm 4.7 \%$ & $.07^{\mathrm{c}}$ & $3.2 \pm 3.5 \%$ \\
& MTR & $4.4 \pm 3.8 \%$ & $3.1 \pm 3.9 \%$ & .56 & $4.2 \pm 3.7 \%$ \\
& T2*WI WM/GM & $3.4 \pm 3.0 \%$ & $2.2 \pm 2.1 \%$ & .37 & $2.6 \pm 2.4 \%$ \\
\hline
\end{tabular}

${ }^{a}$ TRCOV \pm SD is displayed for healthy subjects and those with DCM at rostral (Cl-C3), midcervical (C4-5), or maximally compressed levels in subjects with DCM, and caudal (C6-C7) levels. Sample size was 26 subjects (17 healthy, 9 with DCM) for DTI, 17 subjects (13 healthy, 4 with DCM) for MT, and 16 subjects (5 healthy, 11 with DCM) for T2*Wl.

b Significant differences $(P<.05)$ between pooled TRCOV of metrics at each level.

${ }^{\mathrm{c}}$ Trends $(P<.10)$ in reliability between healthy subjects and those with DCM for each level/metric, and pooled reliability was calculated if no significant differences were found.

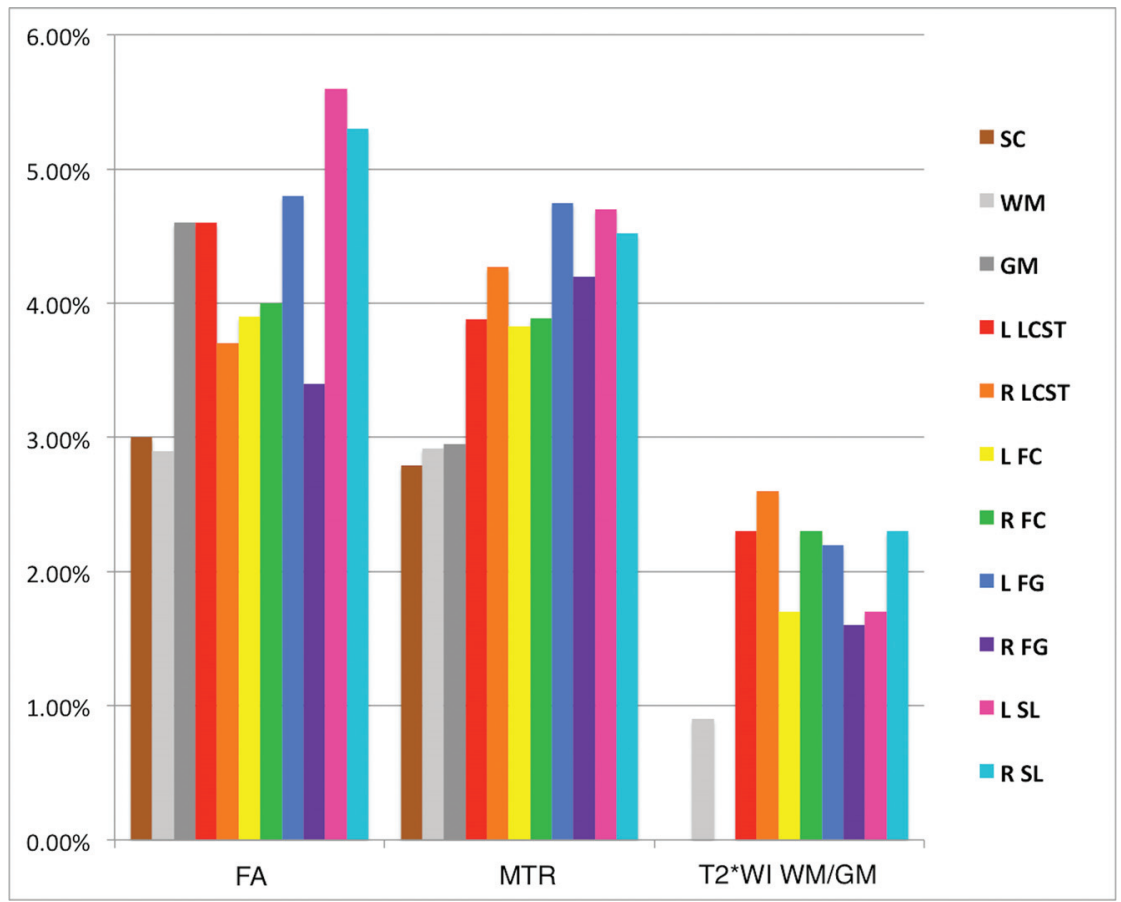

FIG 4. Test-retest coefficients of variation of FA, MTR, and T2*WI WM/GM extracted from SC, WM, GM, and key WM tracts in rostral sections (Cl-C3) are displayed. T2*WI WM/GM ratio shows better reliability than FA and MTR. Metrics derived from the SC and WM show TRCOV $<3 \%$, while GM and key WM tracts show TRCOV $<5 \%$ except for FA of the spinal lemniscus. FC indicates fasciculus cuneatus; FG, fasciculus gracilis; SL, spinal lemniscus; LCST, lateral corticospinal tract; R, right; L, left.

time in addition to anatomic imaging. Image acquisition was successful in all subjects, and automated analysis provided robust readouts from multiple ROIs, with the results validated by acceptable reliability data. Our results establish normative data for CSA, FA, and MTR that are consistent with previous reports at $3 \mathrm{~T},{ }^{12,21,27-29}$ in addition to our novel T2*WI WM/GM metric. T2*WI WM/GM, FA, and MTR all showed strong graywhite contrast and differences between individual WM tracts. FA and MTR showed moderate intersubject and test-retest variability, with similar or better reliability than in previous reports despite differences in acquisition and analysis techniques. ${ }^{8,27-31} \mathrm{~T} 2{ }^{\star}$ WI WM/GM demonstrated low intersubject and test-retest variability, which are favorable statistical prop- erties because they make it more likely that a subject with pathology will show abnormal results (confirmed by encouraging results reported in a companion article $\left.{ }^{20}\right)$. CSA showed greater intersubject variation than other metrics, though this improved slightly following normalization with cervical cord length. Reliability of the CSA measurement was not assessed due to time constraints, but it likely surpasses that of our other measures because it has been previously reported to have TRCOV under $0.5 \%$ by using similar techniques. ${ }^{12}$ Reliability was greatest in the rostral region for all techniques, where healthy subjects and patients with DCM showed similar results. In contrast, patients with DCM showed trends toward diminished reliability at MCLs and caudal levels, likely related to distorted anatomy, increased partial volume effects, increased susceptibility artifacts, and less accurate registration to the SCT template. However, these differences were not significant, and pooled reliability results were all considered acceptable (TRCOV < $5 \%$ ). Our clinically feasible multiparametric approach provides 4 unique quantitative measures in multiple ROIs that reflect aspects of macrostructure and microstructure, with the benefit that these measures cross-validate each other to overcome the limitations (reliability, intersubject variability, sensitivity to pathology) of each individual technique. We anticipate that this multivariate approach can accurately characterize tissue injury in various SC pathologies, which could enable quantitative MR imaging of the SC to achieve clinical translation in the near future.

\section{Normalization for Confounding Factors}

It is essential that quantitative readouts reflect pathologic changes and eliminate confounding effects as much as possible to move toward clinical use of SC quantitative MR imaging. In keeping with prior reports, significant relationships were found between age and $\mathrm{FA}^{5,7,8}$ and MTR, ${ }^{8}$ but not CSA. ${ }^{8,23}$ However, we also identified univariate relationships between MR imaging metrics and sex, height, weight, and cervical cord length, for which we are not aware of previous reports. The relationship between CSA and cervical cord length likely indicates that CSA is related to overall body size because height and weight also showed positive (nonsignificant) correlations. It is unclear why MTR decreases with height, but weak negative trends were also seen with weight and 
Table 5: DTI with and without cardiac triggering ${ }^{a}$

\begin{tabular}{llccc}
\hline Measure & Level & No Triggering & Triggering & $P$ Value \\
\hline FA & Rostral & $0.651 \pm 0.054$ & $0.664 \pm 0.064$ & .41 \\
& Mid/MCL & $0.514 \pm 0.068$ & $0.558 \pm 0.081$ & $.06^{\mathrm{b}}$ \\
\multirow{3}{*}{ TRCOV } & Caudal & $0.534 \pm 0.057$ & $0.562 \pm 0.044$ & $.07^{\mathrm{b}}$ \\
& Rostral & $2.6 \pm 1.9 \%$ & $1.5 \pm 1.2 \%$ & .11 \\
& Mid/MCL & $3.6 \pm 3.6 \%$ & $2.2 \pm 2.3 \%$ & .27 \\
& Caudal & $3.2 \pm 3.5 \%$ & $2.4 \pm 2.3 \%$ & .52 \\
\hline
\end{tabular}

${ }^{a}$ Paired $t$ tests were used to compare FA values extracted from WM at rostral (C1-C3), midcervical (C4-5, healthy subjects), or MCL (subjects with $D C M$ ), and caudal (C6-C7) levels between no triggering vs triggering in 10 subjects (4 healthy, 6 with $D C M)$. Welch $t$ tests were used to compare test-retest coefficient of variation between no triggering $(n=26)$ and triggering $(n=10)$.

${ }^{\mathrm{b}}$ Trends $(P<.10)$.

cervical cord length, suggesting that MTR (reflecting myelin density) is negatively related to overall body size. However, no relationship was present between MTR and CSA in a post hoc test $(r=0.01, P=.94)$. Strong relationships were also found among all 4 metrics and the rostrocaudal level, with the CSA, FA, and MTR showing nonlinearity (Fig 3). CSA increased between the C3 and C6 vertebral levels, reflecting the cervical enlargement that contains increased GM for C5-T1 neurologic levels, and our CSA measurements were highly similar to those in previous reports. $^{32,33}$ WM FA peaked at C2 and locally at C7, where the orientations of axons are almost purely rostrocaudal. In contrast, decreases were seen at C1 (likely due to decussation of corticospinal fibers) and in the cervical enlargement (where a fraction of axons turn and form synapses within the GM). The T2*WI WM/GM ratio was nearly invariant from $\mathrm{C} 1$ to $\mathrm{C} 6$ but increased at $\mathrm{C}$, likely due to increased susceptibility artifacts from the lungs, decreased $\mathrm{SNR}$, and respiratory motion. We suggest a normalization scheme in which CSA, FA, and MTR are linearly corrected for relationships (cervical cord length, age, and age/height, respectively) and all metrics are converted to $z$ scores per rostrocaudal level, as proposed by Uda et $\mathrm{al}^{4}$ for DTI metrics. Although normalization procedures add complexity to data postprocessing, these methods facilitate fair comparisons, decrease nuisance variability, and produce more accurate biomarkers of SC tissue injury.

\section{Quantitative MR Imaging Techniques: Specificity, Accuracy, Feasibility}

The rapidly evolving field of quantitative MR imaging includes a rich array of acquisition techniques, including strict quantitative methods that attempt to measure a specific physical property, such as quantitative MT, longitudinal relaxation rate, and apparent transverse relaxation rate mapping. ${ }^{27,34,35}$ However, such techniques are inherently complex and require specialized pulse sequences, while typically requiring lengthy scan times. Furthermore, these methods face challenges in achieving acceptable SNR and reliability, particularly in the SC, which is considerably more difficult to image than the brain due to magnetic field inhomogeneity and physiologic motion. Similarly, reduced FOV DTI has become available, offering increased SNR and reduced distortions but often requiring increased acquisition times and involving proprietary pulse sequences. ${ }^{31}$ Our protocol purposefully used standard sequences available from all major MR imaging vendors, making it an attractive approach for multicenter studies and clinical use. A recent study comparing reduced FOV with outer volume suppression for cervical SC DTI found only minimal dif- ferences in reliability (intersubject coefficient of variation: reduced $\mathrm{FOV}=$ $3.98 \%$ versus outer volume suppression $=4.59) .{ }^{31}$ Unfortunately, this study did not report $P$ values for these comparisons, and it did not assess intrasubject reliability, but the findings suggest that outer volume suppression provides acceptable reliability.

\section{Cardiac-Triggered DTI}

Previous research suggests that cardiac triggering reduces variance in diffusion time-series by acquiring data during the quiescent phase of cardiac-related SC motion. ${ }^{36}$ However, to our knowledge, no studies have directly compared the test-retest reliability of SC DTI acquisitions with and without cardiac triggering, particularly in the context of multiple acquisitions and outlier rejection during postprocessing. Our pilot data in 10 subjects suggest roughly equivalent results with and without triggering, though trends toward higher FA and lower TRCOV (approximately 1\%) were observed with triggering. Further investigation is needed, but the ungated acquisition used in this study is validated by its acceptable reliability. This simpler approach avoids difficulties with triggering such as variable TR and cardiac irregularities (arrhythmias, tachycardia) that are more common in older or critically ill patients.

\section{Limitations}

Further studies with larger sample sizes would allow greater accuracy for normative data, influences of confounding variables, and differences in DTI with and without cardiac triggering. The normative data are specific to our methodology, and cross-site and cross-vendor validation is required. Our use of automated analysis aimed to reduce bias, but manual editing of segmentations was frequently required. Other DTI metrics were not analyzed due to an a priori decision to focus on FA, due to its consistent results in previous studies. ${ }^{3}$ Our test-retest reliability experiment does not account for scanner drift, but this is unlikely a large source of error because the 2 metrics are ratios rather than absolute signal-intensity values. Neurologically intact subjects with mild SC compression were considered healthy subjects; these changes are evident in $8 \%-26 \%$ of asymptomatic individuals. ${ }^{32,37}$ Moreover, we think that the spectrum of "normal" includes this subgroup, but previous studies have excluded such subjects.

\section{CONCLUSIONS}

Reliable multiparametric assessment of the SC microstructure is possible with standard hardware, acceptable acquisition times, and automated analysis that provide high-fidelity readouts of tissue injury from numerous ROIs. Normalization procedures can be implemented to mitigate confounding effects such as age, height, cervical cord length, and rostrocaudal level, producing more meaningful quantitative metrics. Our clinically suited approach paves the way for translational studies to evaluate potential uses such as improved diagnostics, monitoring of disease progression, and prediction of outcomes.

AJNR Am J Neuroradiol 38:1257-65 Jun 2017 www.ajnr.org 


\section{ACKNOWLEDGMENTS}

This research received funding support from Rick Hansen Institute, as part of the Riluzole in Spinal Cord Injury Study (RISCIS), which is also supported by AOSpine North America, AOSpine International SCI Knowledge Forum, and the North American Clinical Trials Network (NACTN) of the Christopher and Dana Reeve Foundation. This research also received support from the Dezwirek Foundation, the Sherman Clinical Research Unit, and the Gerald and Tootsie Halbert Chair in Spinal Cord Research. Dr. Martin received post-doctoral fellowship support from Canadian Institutes of Health Research.

Disclosures: Allan R. Martin—RELATED: Grant: Rick Hansen Institute, AOSpine North America, North American Clinical Trials Network of the Christopher and Dana Reeve Foundation, the DeZwirek Foundation, the Sherman Clinical Research Unit, and the Gerald and Tootsie Halbert Chair in Spinal Cord Research*; Dr. Martin received post-doctoral Fellowship funding from Canadian Institutes of Health Research (CIHR) that included $\$ 50,000$ (CDN) annual salary support and $\$ 5,000$ annual research allowance that enabled this research. Sukhvinder KalsiRyan-UNRELATED: Consultancy: Neural Outcomes Consulting; Royalties: GRASSP. *Money paid to the institution.

\section{REFERENCES}

1. Wheeler-Kingshott CA, Stroman PW, Schwab JM, et al. The current state-of-the-art of spinal cord: applications. Neuroimage 2014;84: 1082-93 CrossRef Medline

2. Stroman PW, Wheeler-Kingshott C, Bacon M, et al. The current state-of-the-art of spinal cord imaging: methods. Neuroimage 2014; 84:1070-81 CrossRef Medline

3. Martin AR, Aleksanderek I, Cohen-Adad J, et al. Translating stateof-the-art spinal cord MRI techniques to clinical use: a systematic review of clinical studies utilizing DTI, MT, MWF, MRS, and fMRI. Neuroimage Clin 2016;10:192-238 CrossRef Medline

4. Uda T, Takami T, Tsuyuguchi N, et al. Assessment of cervical spondylotic myelopathy using diffusion tensor magnetic resonance imaging parameter at 3.0 Tesla. Spine 2013;38:407-14 CrossRef Medline

5. Mamata H, Jolesz FA, Maier SE. Apparent diffusion coefficient and fractional anisotropy in spinal cord: age and cervical spondylosis-related changes. J Magn Reson Imaging 2005;22:38-43 CrossRef Medline

6. Budzik JF, Balbi V, Le Thuc V, et al. Diffusion tensor imaging and fibre tracking in cervical spondylotic myelopathy. Eur Radiol 2011; 21:426-33 CrossRef Medline

7. von Meyenburg J, Wilm BJ, Weck A, et al. Spinal cord diffusiontensor imaging and motor-evoked potentials in multiple sclerosis patients: microstructural and functional asymmetry. Radiology 2013;267:869-79 CrossRef Medline

8. Taso M, Girard OM, Duhamel G, et al. Tract-specific and age-related variations of the spinal cord microstructure: a multi-parametric MRI study using diffusion tensor imaging (DTI) and inhomogeneous magnetization transfer (ihMT). NMR Biomed 2016;29: 817-32 CrossRef Medline

9. Oh J, Zackowski K, Chen M, et al. Multiparametric MRI correlates of sensorimotor function in the spinal cord in multiple sclerosis. Mult Scler 2013;19:427-35 CrossRef Medline

10. Harrison NA, Cooper E, Dowell NG, et al. Quantitative magnetization transfer imaging as a biomarker for effects of systemic inflammation on the brain. Biol Psychiatry 2015;78:49-57 CrossRef Medline

11. Vavasour IM, Laule $\mathrm{C}$, Li DK, et al. Is the magnetization transfer ratio a marker for myelin in multiple sclerosis? J Magn Reson Imaging 2011;33:713-18 CrossRef Medline

12. Kearney H, Yiannakas MC, Abdel-Aziz K, et al. Improved MRI quantification of spinal cord atrophy in multiple sclerosis. J Magn Reson Imaging 2014;39:617-23 CrossRef Medline

13. Nouri A, Tetreault L, Zamorano JJ, et al. Role of magnetic resonance imaging in predicting surgical outcome in patients with cervical spondylotic myelopathy. Spine 2015;40:171-78 CrossRef Medline
14. Grabher P, Mohammadi S, Trachsler A, et al. Voxel-based analysis of grey and white matter degeneration in cervical spondylotic myelopathy. Sci Rep 2016;6:24636 CrossRef Medline

15. Datta E, Papinutto N, Schlaeger R, et al. Gray matter segmentation of the spinal cord with active contours in MR images. Neuroimage 2017;147:788-99 CrossRef Medline

16. Cohen-Adad J, Buchbinder B, Oaklander AL. Cervical spinal cord injection of epidural corticosteroids: comprehensive longitudinal study including multiparametric magnetic resonance imaging. Pain 2012;153:2292-99 CrossRef Medline

17. Cohen-Adad J, Zhao W, Keil B, et al. 7-T MRI of the spinal cord can detect lateral corticospinal tract abnormality in amyotrophic lateral sclerosis. Muscle Nerve 2013;47:760-62 CrossRef Medline

18. White ML, Zhang Y, Healey K. Cervical spinal cord multiple sclerosis: evaluation with 2D multi-echo recombined gradient echo MR imaging. J Spinal Cord Med 2011;34:93-98 CrossRef Medline

19. Cohen-Adad J. What can we learn from $T 2^{\star}$ maps of the cortex? Neuroimage 2014;93(pt 2):189-200 CrossRef Medline

20. Martin AR, De Leener B, Cohen-Adad J, et al. A novel MRI biomarker of spinal cord white matter injury: $\mathrm{T} 2^{\star}$-weighted white matter to gray matter signal intensity ratio. AJNR Am J Neuroradiol 2017 Apr 20. [Epub ahead of print] CrossRef Medline

21. Cohen-Adad J, El Mendili MM, Lehéricy S, et al. Demyelination and degeneration in the injured human spinal cord detected with diffusion and magnetization transfer MRI. Neuroimage 2011;55:1024-33 CrossRef Medline

22. De Leener B, Lévy S, Dupont SM, et al. SCT: Spinal Cord Toolbox, an open-source software for processing spinal cord MRI data. Neuroimage 2017;145(pt A):24-43 CrossRef Medline

23. Fonov VS, Le Troter A, Taso M, et al. Framework for integrated MRI average of the spinal cord white and gray matter: the MNI-PolyAMU template. Neuroimage 2014;102:817-27 CrossRef Medline

24. Chang LC, Jones DK, Pierpaoli C. RESTORE: robust estimation of tensors by outlier rejection. Magn Reson Med 2005;53:1088-95 CrossRef Medline

25. Asman AJ, Bryan FW, Smith SA, et al. Groupwise multi-atlas segmentation of the spinal cord's internal structure. Med Image Anal 2014;18:460-71 CrossRef Medline

26. Lévy S, Benhamou M, Naaman C, et al. White matter atlas of the human spinal cord with estimation of partial volume effect. Neuroimage 2015;119:262-71 CrossRef Medline

27. Samson RS, Ciccarelli O, Kachramanoglou C, et al. Tissue- and column-specific measurements from multi-parameter mapping of the human cervical spinal cord at 3 T. NMR Biomed 2013;26:1823-30 CrossRef Medline

28. Smith SA, Jones CK, Gifford A, et al. Reproducibility of tract-specific magnetization transfer and diffusion tensor imaging in the cervical spinal cord at 3 Tesla. NMR Biomed 2010;23:207-17 CrossRef Medline

29. Ellingson BM, Salamon N, Grinstead JW, et al. Diffusion tensor imaging predicts functional impairment in mild-to-moderate cervical spondylotic myelopathy. Spine J 2014;14:2589-97 CrossRef Medline

30. Kerkovský M, Bednarik J, Dušek L, et al. Magnetic resonance diffusion tensor imaging in patients with cervical spondylotic spinal cord compression: correlations between clinical and electrophysiological findings. Spine (Phila Pa 1976) 2012;37:48-56 CrossRef Medline

31. Samson RS, Lévy S, Schneider T, et al. ZOOM or non-ZOOM? Assessing spinal cord diffusion tensor imaging protocols for multicentre studies. PLoS One 2016;11:e155557 CrossRef Medline

32. Cadotte DW, Cadotte A, Cohen-Adad J, et al. Characterizing the location of spinal and vertebral levels in the human cervical spinal cord. AJNR Am J Neuroradiol 2015;36:803-10 CrossRef Medline

33. Kato F, Yukawa Y, Suda K, et al. Normal morphology, age-related changes and abnormal findings of the cervical spine, part II: magnetic resonance imaging of over 1,200 asymptomatic subjects. Eur Spine J 2012;21:1499-507 CrossRef Medline 
34. Levesque IR, Giacomini PS, Narayanan S, et al. Quantitative magnetization transfer and myelin water imaging of the evolution of acute multiple sclerosis lesions. Magn Reson Med 2010;63:633-40 CrossRef Medline

35. Freund $\mathrm{P}$, Weiskopf $\mathrm{N}$, Ashburner J, et al. MRI investigation of the sensorimotor cortex and the corticospinal tract after acute spinal cord injury: a prospective longitudinal study. Lancet Neurol 2013; 12:873-81 CrossRef Medline
36. Summers P, Staempfli P, Jaermann T, et al. A preliminary study of the effects of trigger timing on diffusion tensor imaging of the human spinal cord. AJNR Am J Neuroradiol 2006;27:1952-61 Medline

37. Wilson JR, Barry S, Fischer DJ, et al. Frequency, timing, and predictors of neurological dysfunction in the nonmyelopathic patient with cervical spinal cord compression, canal stenosis, and/or ossification of the posterior longitudinal ligament. Spine 2013;38: S37-54 CrossRef Medline 